\title{
Tracing Atmospheric Mercury in the Peruvian Amazon using $\mathrm{Hg}$ Isotopes
}

N. SZPONAR ${ }^{1 *}$, C. VEGA ${ }^{2}$, D. MCLAGAN ${ }^{3}$, J. GERSON $^{4}$, E. BERHARDT ${ }^{4}$, C. MITCHELL ${ }^{3}$, F. WANIA ${ }^{3}$, L.E. FERNANDEZ ${ }^{2}$, AND B.A. BERGQUIST ${ }^{1}$

${ }^{1}$ University of Toronto, Department of Geology, ON, Canada (*correspondence: natalie.szponar@mail.utoronto.ca)

${ }^{2}$ Center for Amazonian Scientific Innovation Wake Forest University, Winston-Salem NC, USA

${ }^{3}$ Universty of Toronto Scarborough, Physical and Environmental Sciences, Toronto, ON, Canada

${ }^{4}$ Duke University, Nicholas School of the Environment, Durham, NC, USA

Artisanal and small-scale gold mining (ASGM) is considered one of the largest contributors of mercury $(\mathrm{Hg})$ emissions and water pollution globally. However, our understanding of $\mathrm{Hg}$ emissions from ASGM and their fate in the environment is not well quantified, with very few atmospheric studies available for the southern hemisphere where ASGM is prevalent.

ASGM activity is expanding in the Madre de Dios (MDD) region of the Peruvian Amazon. Atmospheric gaseous elemental mercury (GEM) samples were collected over 100 sites in MDD including in Puerto Maldonado, a city with a high concentration of gold shops, and along a transect from Puerto Maldonado towards two major gold mining districts. GEM was sampled using novel passive air samplers that allow for sample collection in remote areas and characterization through spatial mapping. Laboratory and field experiments demonstrate that the sampler is reliable for $\mathrm{Hg}$ isotope characterization. Since ore-derived $\mathrm{Hg}$ and $\mathrm{Hg}$ used in gold amalgamation are usually isotopically distinct from typical background atmospheric total gaseous mercury, $\mathrm{Hg}$ isotopes can differentiate ASGM derived sources. GEM concentrations vary widely in the MDD region and are 2 to 4 orders of magnitude higher (up to $5000 \mathrm{ng} / \mathrm{m} 3$ ) than typical background GEM levels in mining districts and areas close to gold shops. Isotopic signatures also vary in the area and are indicative of source. Additionally, $\mathrm{Hg}$ isotopes in soils and vegetation were used to understand the role of rainforest soils in storing $\mathrm{Hg}$ from ASGM versus other regional and global emission sources. This is because GEM uptake by plants and subsequent litterfall is a major $\mathrm{Hg}$ source to forests and soils. Results from Amazonian soils and vegetation collected near ASGM and in remote locations in the context of atmospheric Hg cycling will be discussed. 